\title{
Heat Transfer Enhancement by Vortex Generators for Compact Heat Exchanger of Automobiles
}

\author{
Shigeru Ogawa, Soma Usui \\ Department of Mechanical Engineering, National Institute of Kure College, Kure, Japan \\ Email: ogawa@kure-nct.ac.jp
}

How to cite this paper: Ogawa, S. and Usui, S. (2018) Heat Transfer Enhancement by Vortex Generators for Compact Heat Exchanger of Automobiles. Open Journal of Fluid Dynamics, 8, 321-330. https://doi.org/10.4236/ojfd.2018.83020

Received: August 20, 2018

Accepted: September 17, 2018

Published: September 20, 2018

Copyright $(0) 2018$ by authors and Scientific Research Publishing Inc. This work is licensed under the Creative Commons Attribution International License (CC BY 4.0).

http://creativecommons.org/licenses/by/4.0/

\begin{abstract}
The paper describes the effects of heat transfer enhancement and gas-flow characteristics by wing-type-vortex-generators inside a rectangular gas-flow duct of a plate-fin structure exhaust gas recirculation (EGR) cooler used in a cooled-EGR system. The analyses are conducted using computational fluid dynamics (CFD). The numerical modelling is designed as a gas-flow rectangular duct of an EGR cooler using two fluids with high temperature gas and coolant water whose flow directions are opposite. The gas-flow duct used to separate two fluids is assembled with a stainless steel material. The inlet temperature and velocity of gas flowed inside gas-flow duct are $400^{\circ} \mathrm{C}$ and 30 $\mathrm{m} / \mathrm{s}$, respectively. Coolant water is flowed into two ducts on both a top and a bottom surface of the gas-flow duct, and the inlet temperature and velocity is $80^{\circ} \mathrm{C}$ and $0.6 \mathrm{~m} / \mathrm{s}$, respectively. Wing-type-vortex-generators are designed to achieve good cooling performance and low pressure drop and positioned at the center of the gas-flow duct with angle of inclination from 30 to 150 degrees at every 15 degrees. The temperature distributions and velocity vectors gained from numerical results were compared, and discussed. As a result, it is found that the vortices guided in the proximity of heat transfer surfaces play an important role in the heat transfer enhancement and low pressure drop. The collapse of the vortices is caused by complicated flow induced in the corner constituted by two surfaces inside gas-flow duct.
\end{abstract}

\section{Keywords}

Exhaust Gas Recirculation (EGR), Vortex Generator, Thermal Fluid Analysis, Longitudinal Vortex

\section{Introduction}

Exhaust gas recirculation (EGR) has been recently focused on enhancement of 
fuel consumption and reduction of nitrogen oxides in small type of internal combustion engines for automobiles. Figure 1 indicates schematic view of EGR cooling system of automobiles. In particular, EGR coolers to cool down exhaust gases are regarded as one of main parts which determine the performance of EGR system as well as layouts in engine rooms. For that reason it is indispensable to achieve the cooling performance and the compactness simultaneously. Structures of EGR coolers consist mainly of Shell-and-Tube types and Plate-Fin types as shown in Figure 2. In general, the former has advantage of cost performance whereas the latter has high cooling performance per unit volume. Some products [1] [2] [3] of EGR coolers of Plate-Fin types are seeking for cooling performances by inner fins enlarging heat transfer areas and by longitudinal vortex generators. It is known from previous studies [4]-[9] that heat transfer of heat exchangers with longitudinal vortices is very efficiently performed by enhancement of convection heat transfer from main flows toward boundary layers and by longitudinal vortices which make great contributions to weakening boundary layers. Longitudinal vortices are also expected to keep effects of heat transfer enhanced with low pressure loss by maintaining their persistence property in the flow direction. In the previous studies, however, there are few papers on heat transfer in the square duct although most of the papers are discussed on effects of convective heat transfer on the plate. The author has so far been seeking for basic understanding of heat transfer to obtain high cooling performance [10] [11]. Our final goal of the study is to propose inner structures for EGR coolers which can make cooling performances and compact lightweight compatible. The paper aims to clarify not only effects of a vortex generator on flow fields by numerically changing inclination angles of the delta and rectangular wings, but also the relationships between longitudinal vortices and effects of enhancement of heat transfer.

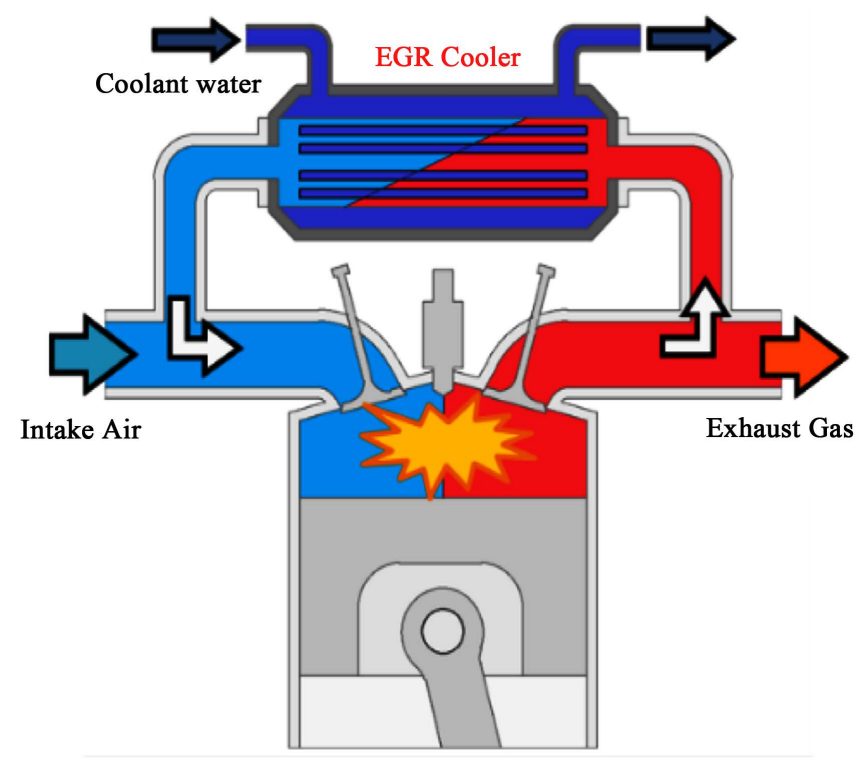

Figure 1. EGR cooling system. 


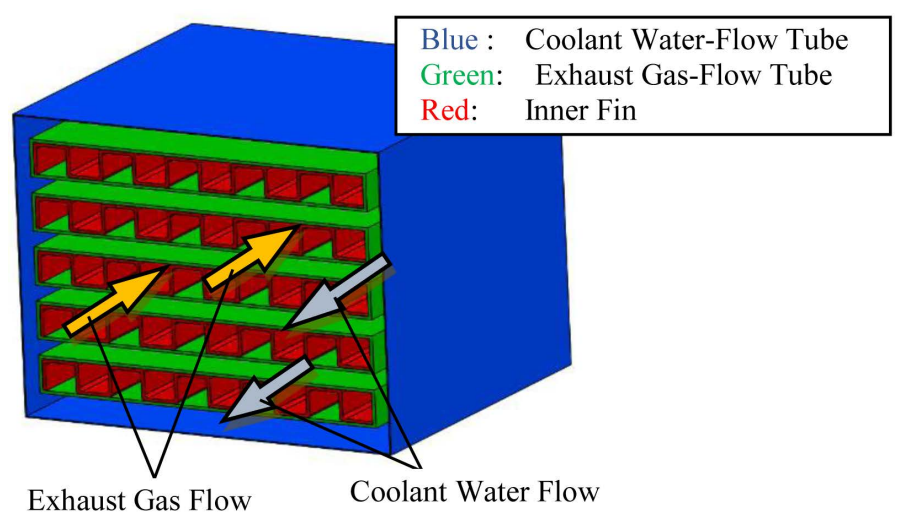

Figure 2. Counter-flow plate-fin type EGR cooler.

\section{Numerical Method}

The numerical simulation was conducted with the heat-fluid-analysis software, STAR-CCM+ of CD-adapco.

\subsection{Heat Exchange Duct Model}

Figure 3 shows the analytical model of the heat exchanger which models EGR cooler with one duct. The gas-flow duct is a square with $H=2.2 \mathrm{~mm}$ and $L=60$ $\mathrm{mm}$ where a vortex generator was immersed at the center of the square duct. The separated wall made of steel is $0.35 \mathrm{~mm}$ thick. The inlet temperature and velocity of hot gas flowed into gas-flow duct are $400^{\circ} \mathrm{C}$ and $30 \mathrm{~m} / \mathrm{s}$, respectively. The direction of gas flows is defined as flow direction. On the other hand, coolant water is oppositely flowed into two ducts which are attached to the upper and lower surfaces of the gas-flow duct, and the inlet temperature and velocity of coolant water are $80^{\circ} \mathrm{C}$ and $0.6 \mathrm{~m} / \mathrm{s}$, respectively.

\subsection{Thermal Fluid Analysis}

In order to obtain the solution, three equations were used. The Navier-Stokes equation is used as RANS (Reynolds Averaged Navier-Stokes Simulation) with turbulent models. Two other equations are equation of continuity and energy equation. As turbulent vortex viscosity models, K- $\varepsilon$ model was used since it has advantage of better convergence in calculation and is frequently used for numerical analyses of EGR coolers. Maximum step numbers in steady analyses and total mesh numbers are 450 and about 800,000, respectively. Polyhedral meshes with 10 to 15 planes provided by Star-CCM+ were employed as mesh shapes to highly enhance the numerical accuracy and convergence. Prism layer was applied to reproduce behaviors of boundary layer in the vicinity of the heat transfer plane. The prism layer was divided into 10 layers and its total thickness amounts to 0.1 .

\section{Flow Characteristics of a Single Vortex Generator}

In order to clarify characteristics of a single vortex generator, thermal fluid 


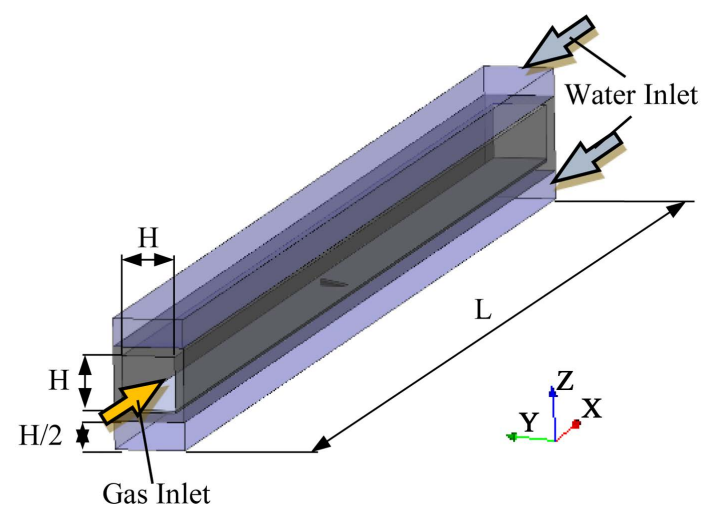

Figure 3. Analytical model.

analysis investigated the flow and heat transfer by changing angles of inclination with respect to a delta wing and a rectangular wing.

\subsection{Vortex Generator Model}

The shape of the vortex generator is an isosceles triangle with each side of $0.5 \mathrm{H}$ and height $H$ and a rectangular wing with each side of $0.4 H$ and $0.8 H$. The set-up condition is defined in such a way that $X$ and $Y$ axis are the gas flow direction and the rotating axis, respectively and angles of inclination $\psi$ are angles between $X$ axis and the delta wing. Angles of inclination $\psi$ were parametrically studied by six different angles inclined to the flow direction, with the backward inclination ( $\psi=30,45,60$ degrees) and forward inclination $(\psi=120,135,150$ degrees) as shown in Figure 4. Table 1 indicates the percentage of projected area of the generator in the flow direction to the section area of the gas-flow duct. The percentages of projected area of the vortex generator range from 13\% to $28 \%$ almost equivalent to those of commercial EGR cooler.

\subsection{Effect of Angles of Inclination for a Single Vortex Generator}

Figure 5 indicates gas outlet temperature and pressure drop, changing angles of inclination for a single vortex generator. It is found that delta and rectangle show the similar tendency, and that at the same angles the delta has better performance than the rectangle in that gas outlet temperature and pressure drop of the delta wing are lower than those of the rectangular wing. Furthermore, compared with wings with the same angles of inclination, gas outlet temperatures of wings with forward inclination tend to be lower than those of wings with backward inclination whereas pressure drops are almost the same. Therefore, it is considered that whether the wings incline forward or backward greatly affects the flow field behind the wings. Figure 6 shows streamlines behind the delta wings. It is found that there exist twin longitudinal vortices. Figure 7 depicts temperature distributions and velocity vectors which shows great differences of rotational directions. The forward inclined wings generate downwash whereas the backward ones induce upward flow. The strength of vorticity is expressed by vorticity vector. Vorticity $\omega$ is mathematically defined as the rotation or curl of 


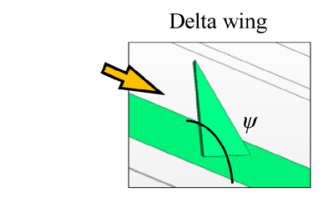

(a)

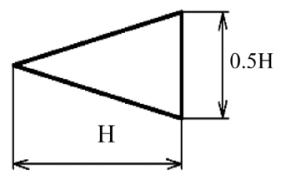

(b)

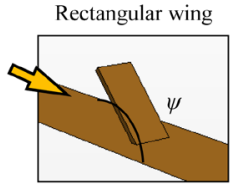

(c)

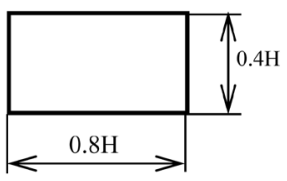

(d)

$H$ : Height and width of the duct.

Figure 4. Dimensions of vortex generator. (a), (b) show delta wing, and (c), (d) rectangular wing.

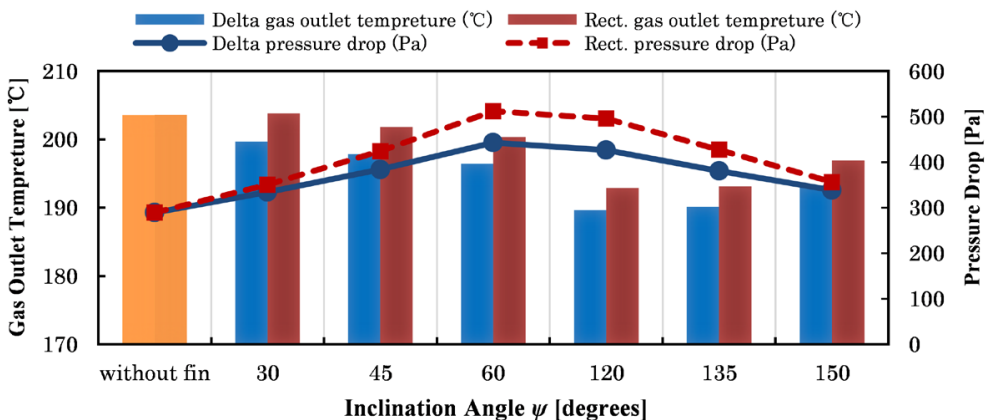

Figure 5. Gas outlet temperature and pressure drop for a single vortex generator.

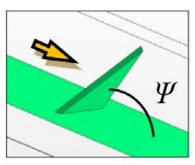

$\Psi=45$ degrees

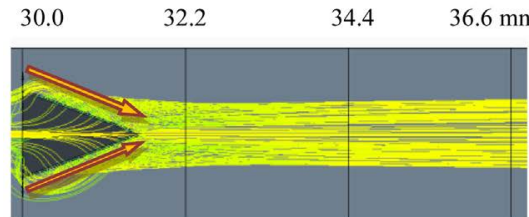

(a)

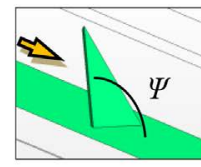

$\Psi=135$ degrees

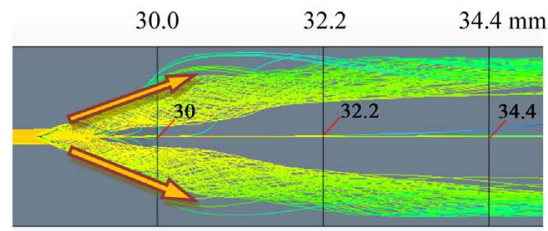

(b)

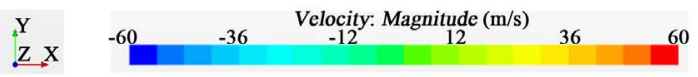

Figure 6. Streamlines behind delta wings at $\psi=45^{\circ}$ and $135^{\circ}$. (a) Streamlines; (b) Streamlines.

Table 1. Percentage of projected areas for the delta wing and rectangular wing.

\begin{tabular}{ccc}
\hline $\begin{array}{c}\text { Angles of inclination } \\
\Psi \text { [degree] }\end{array}$ & \multicolumn{2}{c}{ Projected area of vortex generators [\%] } \\
\cline { 2 - 3 } 30,150 & Delta Wing & Rectangular Wing \\
45,135 & 13 & 16 \\
60,120 & 18 & 23 \\
\hline
\end{tabular}




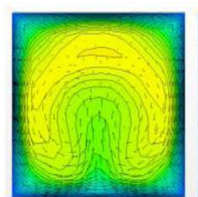

$32.2 \mathrm{~mm}$

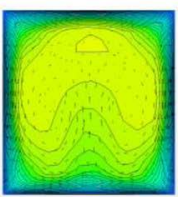

$34.4 \mathrm{~mm}$

(a)

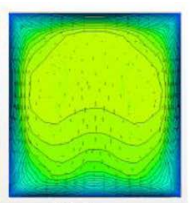

$36.6 \mathrm{~mm}$

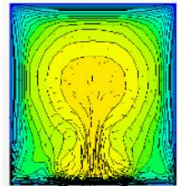

$30.0 \mathrm{~mm}$

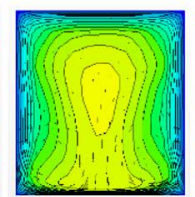

$32.2 \mathrm{~mm}$

(b)

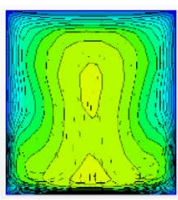

$34.4 \mathrm{~mm}$

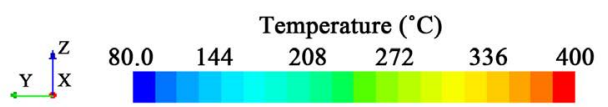

Figure 7. Temperature distributions and velocity vectors for delta wings at $\psi=45^{\circ}$ and $135^{\circ}$. (a) Delta wing $\psi=45^{\circ}$ (Backward); (b) Delta wing $\psi=135^{\circ}$ (Forward).

velocity vectors described by Equation (1) and the x component of vorticity $\omega_{\mathrm{x}}$ by Equation (2). Here $\omega_{x}$ indicates the rotation of fluid particles on the plane normal to velocity $u$ in the downstream direction.

$$
\begin{gathered}
\boldsymbol{\omega}=\nabla \times \boldsymbol{U}=\boldsymbol{r o t} \boldsymbol{U}=\text { curl } \boldsymbol{U} \\
\omega_{x}=\frac{\partial w}{\partial Y}-\frac{\partial v}{\partial Z} \\
\omega_{y}=\frac{\partial u}{\partial Z}-\frac{\partial w}{\partial X} \\
\omega_{z}=\frac{\partial v}{\partial X}-\frac{\partial u}{\partial Y}
\end{gathered}
$$

where $U(u, v, w)$ is velocity vector, $\mathrm{w}$ transverse velocity, $v$ spanwise velocity, and $\omega\left(\omega_{x}, \omega_{y}, \omega_{z}\right)$ vorticity. Figure 8 depicts the vorticity evaluated by Equation (2). It is found that the delta wing with $\psi=135^{\circ}$ has much stronger vorticity than that with $\psi=45^{\circ}$. The vorticity distributions show that twin vortices with opposite rotational directions are generated behind the leading edge of the delta wing. The forward inclined delta wing induces strong vorticities $\omega_{\mathrm{x}}$ rotating around the flow direction, which are called longitudinal vortices. The longitudinal vortices are rotating along the surface of the delta wing from the tip angle position down toward the heat transfer plane at the bottom of the delta wing. This is because the flow separation caused at the tip angle of the wing generates downwash owing to the forward inclination of the wing. As a result, temperature distributions show that great change of the temperature occurs extremely close to the heat transfer plane. The vortices cause the downwash to convey the hot gas at the center of the duct down toward the heat transfer plane where convective heat transfer is enhanced.

\section{Heat Transfer Characteristics of a Group of Vortex Generators}

This section describes flow field and heat characteristics induced by a group of vortex generators, changing the array of vortex generators. As the configuration parameters of the vortex generators, six arrays of the vortex generators were totally evaluated. Four arrays are delta wings or rectangular ones of setting the 
same inclined angles with $\psi=45^{\circ}$ or $135^{\circ}$ respectively. Other two arrays are delta wings or rectangular ones of arranging $\psi=45^{\circ}$ and $135^{\circ}$ alternately. The pitch $P$ between the wings are the same distance as $P=1.5 \mathrm{H}$, where $H$ is the height of gas duct. The total number of generators is 18 . These groups of vortex generators were investigated, setting in the center of the gas duct. Figure 9 indicates gas outlet temperature and pressure drop by changing the arrays of generators. Compared to delta and angular wings with the same angle of inclination, gas outlet temperature of rectangular wings is lower than that of delta wings whereas pressure drop of delta wings is smaller than that of rectangular wings. Gas outlet temperature of delta and rectangular types of wings with $\psi=135^{\circ}$ is the lowest, and that of wings with $\psi=45^{\circ}$ and wings with $\psi=45^{\circ}$ and $135^{\circ}$ alternately are almost the same. Concerning pressure drop, the array of wings with $\psi=135^{\circ}$ has the largest one and the array of wings with $\psi=45^{\circ}$ and $135^{\circ}$ alternately has the lowest one. In order to clarify the mechanism, vorticity distributions were investigated at the six sections located at 23.4, 25.6, 27.8, 30.0, 32.2, and $34.4 \mathrm{~mm}$ from the gas inflow entrance. Of six numerical results, typical four vorticity distributions are shown in Figure 10 for delta and rectangular wings with $\psi=135^{\circ}$, and with $\psi=45^{\circ}$ and $135^{\circ}$ alternately. The arrays of vortex generators with $\psi=$ $135^{\circ}$ generate strong longitudinal vortices which continue to remain in the

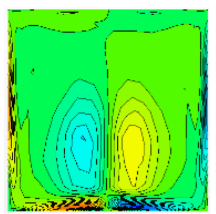

$32.2 \mathrm{~mm}$

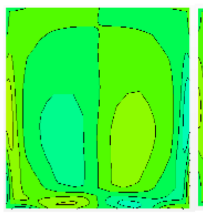

$34.4 \mathrm{~mm}$

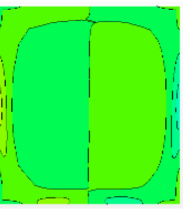

36.6

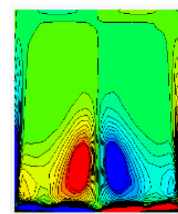

$30.0 \mathrm{~mm}$

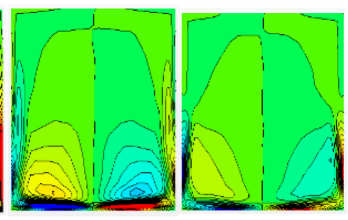

$32.2 \mathrm{~mm}$

$34.4 \mathrm{~mm}$

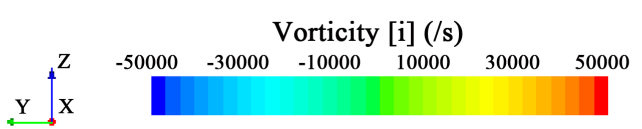

Figure 8. Vorticity distributions of $\omega_{x}$ for delta wing at $\psi=45^{\circ}$ and $135^{\circ}$. (a) Delta Wing $\psi=45^{\circ}$ (Backward); (b) Delta Wing $\psi=135^{\circ}$ (Forward).

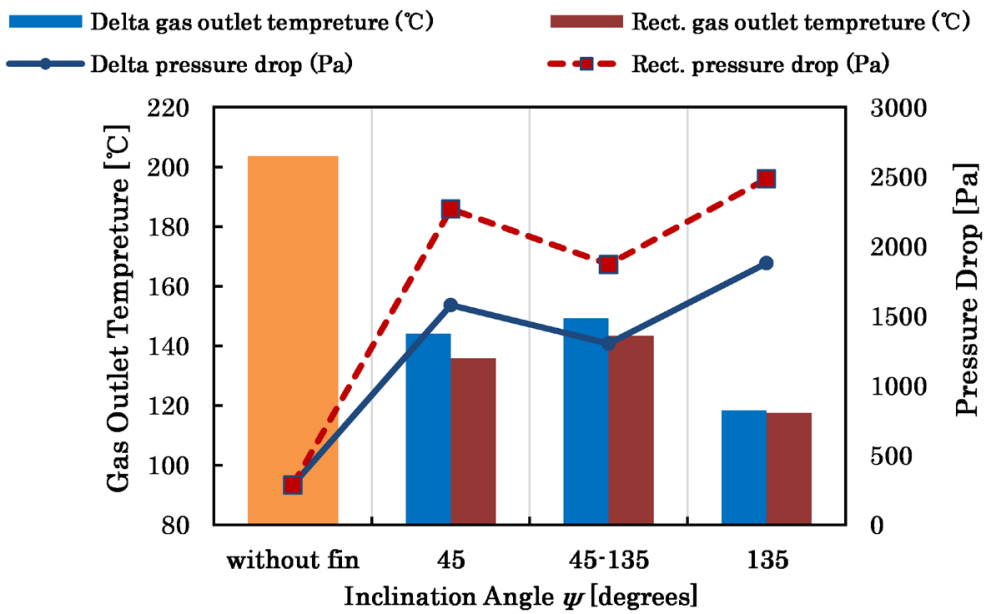

Figure 9. Gas outlet temperature and pressure drop for a group of vortex generators. 


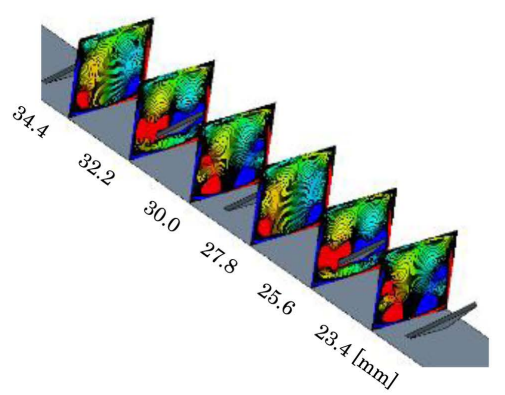

(a)

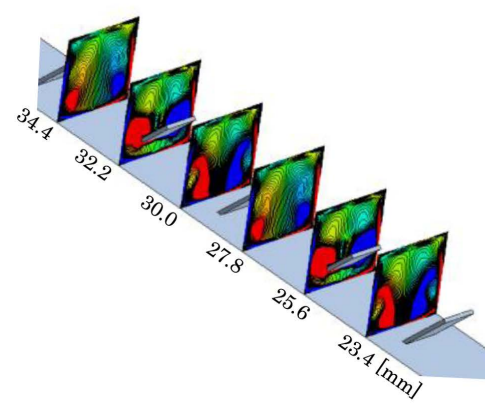

(c)

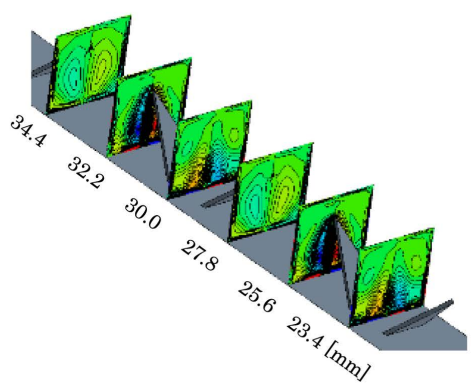

(b)

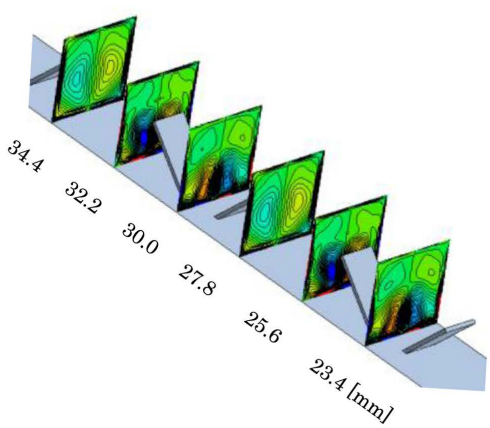

(d)

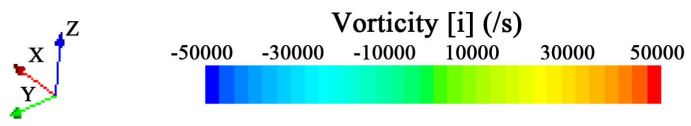

Figure 10. Vorticity distributions for a group of vortex generators. (a) Delta $\left(\Psi=135^{\circ}\right)$; (b) Delta $\left(\Psi=45^{\circ}, 135^{\circ}\right.$ alternately); (c) Rec. $\left(\Psi=135^{\circ}\right)$; (d) Rec. $\left(\Psi=45^{\circ}, 135^{\circ}\right.$ alternately).

downstream direction. This leads to the high enhancement of heat transfer. Regarding pressure drop, there simultaneously exist two vortices generated from an upstream generator and a downstream one. The vortices rotating in the same direction interact each other and at the contact plane they have the opposite velocity vectors, which induce the high pressure drop. As a result, it follows that delta and rectangular wings with $\psi=135^{\circ}$ have high cooling performance, but pressure drop is also high. Delta and rectangular wings with $\psi=45^{\circ}$ and $135^{\circ}$ alternately generate the weakest vorticity distributions. This is because the vortices generated by backward inclined wings and forward inclined wings rotate each other in opposite direction, which is considered to weaken the strength of vorticity and leads to lowest pressure loss.

\section{Conclusions}

The paper numerically studied the enhancement of heat transfer and array conditions for a single generator as well as a group of the delta and rectangular wings in one duct. As a result, the following were concluded.

1) Whether vortex generators are inclined backward or forward toward the 
flow direction makes a significant difference in the flow field. Forward inclined vortex generators induce the twin strong longitudinal vortices behind the generators. The longitudinal vortices induce the mainstream with hot gas toward the heat transfer plane to enhance the heat transfer efficiently. A delta and a rectangular wing show the similar trend.

2) A group of delta and rectangular wings with $\psi=135^{\circ}$ have high cooling performance, but pressure drop is also high. Delta and rectangular wings with $\psi$ $=45^{\circ}$ and $135^{\circ}$ alternately generate the weakest vorticity distributions. This is because the vortices generated by wings with backward inclination and wings with forward inclination rotate each other in opposite direction.

\section{Acknowledgements}

This work has been supported by Japan Grant-in-Aid for Scientific Research (C) under contract No. 17K06174.

\section{Conflicts of Interest}

The authors declare no conflicts of interest regarding the publication of this paper.

\section{References}

[1] Iwasaki, M., Hara, J. and Honda, I. (2014) Development of Vortex Generator for EGR Cooler. FISITA, 89-94.

[2] Hiramatsu, M., Ishimaru, T. and Ohkouchi, T. (1991) Numerical Analysis on Inner-Fins for Intercoolers. Transactions of the Japan Society of Mechanical Engineers, Series B, 57, 176-182. (In Japanese)

[3] Ozawa, T. (2002) Development of EGR Cooler. Calsonic Kansei World, 37-43. (In Japanese)

[4] Fiebig, M., Kallweit, P., Mitra, N. and Tiggelbeck, S. (1991) Heat Transfer Enhancement and Drag by longitudinal Vortex Generators in Channel Flow. Experimental Thermal and Fluid Science, 4, 103-114. https://doi.org/10.1016/0894-1777(91)90024-L

[5] Tiggelbeck, S., Mitra, N. and Fiebig, M. (1992) Flow Structure and Heat Transfer in a Channel with Multiple Longitudinal Vortex Generators. Experimental Thermal and Fluid Science, 5, 425-436. https://doi.org/10.1016/0894-1777(92)90029-5

[6] Jacobi, A.M. and Shah, R.K. (1995) Heat Transfer Surface Enhancement through the Use of Longitudinal Vortices: A Review of Recent Progress. Experimental Thermal and Fluid Science, 11, 295-309. https://doi.org/10.1016/0894-1777(95)00066-U

[7] Itoh, M., Tanaka, T. and Matsushima, H. (2002) Performance Evaluation of a Heat Exchanger with Vortex Generators for Air-Conditioners: Influence of Vortex-Generator Height. Transactions of the Japan Society of Mechanical Engineers, Series B, 68, 188-193. (In Japanese) https://doi.org/10.1299/kikaib.68.188

[8] Chen, Y., Fiebig, M. and Mitra, N.K. (2000) Heat Transfer Enhancement of Finned Oval Tubes with Staggered Punched Longitudinal Vortex Generators. International Journal of Heat and Mass Transfer, 43, 417-435. https://doi.org/10.1016/S0017-9310(99)00157-X 
[9] Torii, K., Nishino, K. and Nakayama, K. (1994) Mechanism of Heat-Transfer Enhancement by Longitudinal Vortices in a Flat Plate Boundary Layer. Transactions of the Japan Society of Mechanical Engineers, Series B, 60, 275-282. (In Japanese) https://doi.org/10.1299/kikaib.60.997

[10] Ogawa, S., Usui, S., Akaishi, I., Takeda, J. and Yano, K. (2015) Study on Optimal Counter-Flow for High Heat Transfer. Proceedings of Thermal Engineering Conference 2015, Osaka, 24 October 2015, USB Proceedings No. 15-48, Paper No. C 141.

[11] Ogawa, S., Usui, S. and Akaishi, I. (2016) Thermal Fluid Analysis on Heat Transfer Enhancement for Heat Exchanger. Proceedings of the $4^{\text {th }}$ International Forum on Heat Transfer, IFHT2016, Sendai, 2-4 November 2016, Paper No. IFHT2016-1916. 